\title{
IUFOST2006/142 Sequence-specific enzymes for totally new food ingredients
}

\author{
L. Van Orsouw \\ DIREVO Biotech AG, Nattermannallee 1, 50829 Cologne, Germany \\ vorsouw@direvo.de
}

There is a steadily rising demand for bioactive peptides as functional food ingredients, as well as for the avoidance of anti-nutritional factors. Direvo addresses this demand for improved functional foods, using two powerful technologies:

a) Direvo's application-relevant, high-output Directed Evolution System for enzymes b) Creation of totally new products by engineering custom-order, sequence-specific proteases that can create or remove any peptide or protein in functional food formulations (our proprietary "NBEC)"-technology) Direvo has already selected a number of targets for which the NBE technology can provide a solution. One of these targets is angiotensin II, a common cause of hypertension. This potent bioactive peptide is formed by the angiotensin-converting enzyme (ACE). ACE inhibitors thus represent a validated means to reduce the generation of angiotensin II. Direvo announces the successful application of the NBEC technology to obtain ACE inhibitors using new enzymes that specifically cut ACE inhibitory peptides from bulk proteins already used as common food ingredients. Direvo's proprietary NBE(C) technology has already been used to create an enzyme that inactivates TNFalpha, another causative agent in chronic human diseases. Successful animal studies performed in 2005 using this enzyme underline the breadth and impact of this novel technology. In the development of NBE(C-derived ACE inhibitors, Direvo had the choice between multiple approaches. One of them was to screen for NBE(C) proteases that will cut a known peptide sequence from a given substrate (bulk protein). Such peptides have been described in literature and Direvo has successfully targeted pre-selected peptide sequences. For this project, however, the decision was made to develop a screen based on ACE inhibition, in order to select even better inhibitors. How does Direvo make enzymes with a specificity that does not occur in nature? First, we select suitable enzyme scaffolds: a lipase for lipid substrates, a hemicellulase for carbohydrate substrates, or, as in this case, a protease for a protein or peptide substrates. Using our proprietary approach, we prepare NBEC protease libraries containing millions of variations in the Specificity Determining Regions. Each of these NBE@ proteases have a different specificity. These libraries are then screened at a rate of up to a million different NBEC proteases per day, using the predictive screen developed for this project. We finally selected the best NBEC proteases that generated the most potent ACE-inhibitors from the bulk food ingredient. The NBEC technology can be applied to a wide range of different applications which will be discussed. 